benötigt. Damals hat es ähnlich lange gedauert, bis die aus dem Nationalsozialismus übernommenen leitenden Beamten durch Angehörige der Nachkriegsgeneration ersetzt werden konnten. Insofern ist der Transformationsprozess in den neuen Bundesländern personell noch nicht abgeschlossen. Die Entwicklung seit 1990 deutet aber darauf hin, dass bei der Rekrutierung von Spitzenbeamten in Ostdeutschland zunehmend weniger auf „Direktimporte“ aus den alten Bundesländern zurückgegriffen wird, so dass die Existenz einer „Betondecke aus leitenden Beamten mit westdeutschem Hintergrund“ 42 ein historisches Durchgangsstadium sein dürfte.

42 Dieter Schimanke, Dilemmata der Personalpolitik, in: Hans-Ulrich Derlien (Hrsg.), Zehn Jahre Verwaltungsaufbau Ost - eine Evaluation, Baden-Baden 2001, S. 179 - 187, S. 182.

\title{
Volksgesetzgebung in den deutschen Bundesländern: ein Vorschlag zu ihrer empirischen Erfassung aus subnational- vergleichender Perspektive*
}

\author{
Christina Eder und Raphael Magin
}

Seit Beginn der 1990er Jahre kam es in den deutschen Ländern zu einem Ausbau der unmittelbaren Volksrechte sowie zu einer beträchtlichen Zunahme der direktdemokratischen Praxis. ${ }^{1}$ Als Folge dieser Entwicklung steht die Volksgesetzgebung den Bürgern auf Landesebene nunmehr flächendeckend zur Verfügung. Unter dem Begriff „Volksgesetzgebung“ wird hier die institutionalisierte Form der direkten Bürgerbeteiligung an der Gesetzgebung verstanden, wobei sowohl die Eröffnung des Verfahrens als auch die Formulierung der Vorlage den Bürgern selbst vorbehalten ist. Diese „bottom-up-Beteiligungsrechte“ sind mittlerweile in allen Bundesländern vorgesehen und weisen mit großem Abstand die häufigste Nutzung aller direktdemokratischen Instrumente auf. ${ }^{2}$ Die übrigen Instrumente der direkten Demokratie (fakultatives Referendum, obligatorisches Verfassungsreferendum, Abberufung des Landtages, Plebiszit) sind nicht flächendeckend verbreitet und werden nur selten

Der vorliegende Beitrag wurde im Rahmen des von der Deutschen Forschungsgemeinschaft finanzierten Forschungsprojekts „Demokratiemuster in den deutschen Bundesländern und ihre politischen, gesellschaftlichen und ökonomischen Auswirkungen “ verfasst. Eine frühere Version des Papiers wurde auf dem Jahreskongress der Schweizerischen Vereinigung für Politikwissenschaft 2006 präsentiert. Wir danken Markus Freitag und Adrian Vatter und den Gutachtern der Zeitschrift für Parlamentsfragen für ihre Anregungen und Kommentare sowie David Fritz und Julia Karst für ihre Mithilfe bei Recherchen und Korrekturen.

1 Siehe Bärbel Weixner, Direkte Demokratie in den Bundesländern, in: APuZ, B 10 (2006), S. 18 - 24.

2 Siehe Raphael Magin / Christina Eder / Adrian Vatter, Direkte Demokratie in den Bundesländern. Ein Vergleich der Institutionen und Anwendungsmuster, in: Achim Hildebrandt / Frieder Wolf (Hrsg.), Politik in den Bundesländern, Wiesbaden 2008, S. 345 - 364; Frank Rehmet, Direkte Demokratie in den deutschen Bundesländern, in: Theo Schiller / Volker Mittendorf(Hrsg.), Direkte Demokratie. Forschung und Perspektiven, Wiesbaden 2002, S. $102-114$. 
oder gar nicht angewandt. Auch die Abstimmungen zur Verabschiedung der Landesverfassungen in den ostdeutschen Bundesländern bleiben hier bewusst ausgeklammert. ${ }^{3}$

\section{Forschungstand}

Der kontinuierliche Ausbau und die stetig wachsende Popularität der Volksmitsprache in den Bundesländern schlägt sich in einer verstärkten politikwissenschaftlichen Analyse nieder: In einem von Andreas Kost ${ }^{4}$ edierten Sammelband ist jedem Bundesland ein Kapitel gewidmet, in dem sowohl das Prozedere als auch die Praxis der unmittelbaren Volksbeteiligung dokumentiert werden; Frank Rehmet ${ }^{5}$ präsentiert einen kurzen Vergleich der Verfahren und Anwendungsfälle der direkten Demokratie in den Bundesländern, und Bärbel Weixner $^{6}$ bietet einen umfassenden Überblick über die Verfahrensausgestaltung in den einzelnen Ländern sowie die Anwendungspraxis, wobei besonders thematische Schwerpunkte Beachtung finden. Auch wurden einige Sammelbände, Einführungen und Zeitschriftenbeiträge zur unmittelbaren Bürgerbeteiligung veröffentlicht, die unter anderem die direkte Demokratie auf Landesebene beleuchten. ${ }^{7}$

Obwohl diese Beiträge die einzelnen Verfahrenselemente der Volksgesetzgebung ausführlich beschreiben, mangelt es bislang an Arbeiten, welche die Informationen zu den direktdemokratischen Instrumenten in den Bundesländern bündeln und in systematisch vergleichender Perspektive operationalisieren. Ziel dieses Beitrages ist daher eine analytisch-vergleichende Erfassung und Messung der Volksgesetzgebung in den deutschen Bundesländern. Der resultierende Index soll Aufschluss darüber geben, wie einfach oder schwierig es für die Bürger ist, sich mittels der Volksgesetzgebung in den politischen Entscheidungsprozess einzubringen. Es geht also um die relative Höhe der Verfahrenshürden im Bundesländervergleich, welche die Antragsteller zu überwinden haben. Bei niedrigen Hürden soll im Folgenden von einem offenen, bei hohen Hürden von einem geschlossenen Verfahren gesprochen werden.

Die bislang einzigen systematischen Messversuche der Offenheit im Bundesländervergleich stammen vom Verein „Mehr Demokratie e.V.“8. In diesen „Volksentscheid-Ran-

3 Siehe dazu Raphael Magin / Christina Eder, Direkte Demokratie in den Bundesländern: Grundlagen, Institutionen, Anwendungen, in: Markus Freitag / Uwe Wagschal (Hrsg.), Direkte Demokratie. Bestandsaufnahmen und Wirkungen im internationalen Vergleich, Münster 2007, S. 151 - 187.

4 Andreas Kost (Hrsg.), Direkte Demokratie in den deutschen Ländern. Eine Einführung, Wiesbaden 2005.

5 Frank Rehmet, a.a.O.

6 Bärbel Weixner, Direkte Demokratie in den Bundesländern. Verfassungsrechtlicher und empirischer Befund aus politikwissenschaftlicher Sicht, Opladen 2002.

7 Siehe Hermann K. Heußner / Otmar Jung, Mehr direkte Demokratie wagen. Volksbegehren und Volksentscheid: Geschichte - Praxis - Vorschläge, München 1999; Peter Massing, Direkte Demokratie - eine Einführung, Schwalbach/Ts. 2005; Theo Schiller, Direkte Demokratie. Eine Einführung, Frankfurt am Main 2002; Theo Schiller / Volker Mittendorf (Hrsg.), Direkte Demokratie. Forschung und Perspektiven, Wiesbaden 2002; Otmar Jung, Daten zu Volksentscheiden in Deutschland auf Landesebene, in: ZParl, 24. Jg. (1993), H. 1, S. 5 - 13.; ders., Direkte Demokratie: Forschungsstand und Forschungsaufgaben 1995, in: ZParl, 26. Jg. (1995), H. 4, S. 658 -677 .

8 Ralph Kampwirth, Erstes Volksentscheid-Ranking. Die direktdemokratischen Verfahren der Länder und Gemeinden im Vergleich, Berlin 2003; Frank Rehmet / Karin Flothmann / Tim Weber, Zweites Volksentscheid-Ranking. Länder und Gemeinden im Demokratievergleich, Berlin 2007. 
kings“" werden verschiedene Teilaspekte der direkten Demokratie betrachtet und Schulnoten zwischen 1,0 (sehr gut) und 6,0 (ungenügend) für den Grad der Offenheit vergeben. Trotz einer sehr ausführlichen Darstellung der wichtigsten Entwicklungen und zahlreicher Verfahrenselemente ist die Messung jedoch an mehreren Stellen mit Mängeln behaftet:

(1) Die Darstellung ist unvollständig. So werden Bekanntmachungsregeln nur für Volksentscheide berücksichtigt und auch dort nur hinsichtlich des Vorhandenseins einer Abstimmungsbroschüre. Für die Einleitungs- und die Volksbegehrensphase fehlt eine entsprechende Darstellung gänzlich. ${ }^{\text {? }}$

(2) Ebenso verhält es sich mit der gerichtlichen Überprüfung abgelehnter Initiativen; auch dieses Merkmal ist nicht Bestandteil der Rankings.

(3) Weiterhin wird bei der Kostenerstattung nur das Vorhandensein des Merkmals untersucht. Die mitunter enormen Unterschiede im Umfang wie auch der Zeitpunkt der Erstattung werden vernachlässigt. ${ }^{10}$

(4) Mit der Intensität der Reformdiskussion in den einzelnen Bundesländern wurde ein Indikator berücksichtigt, zu dessen Operationalisierung keine genauen Angaben gemacht werden. ${ }^{11}$

(5) Weiterhin ist die Rechtsprechungspraxis zur direkten Demokratie eingeflossen, was für einige Bundesländer zur Auf- beziehungsweise Abwertung im Ranking führte. Wie die Bewertung dieses Aspektes im Detail zustande kommt, ist dabei nicht eindeutig ersichtlich. ${ }^{12}$

(6) Auch die übrige Praxis der Wertevergabe erweist sich als nicht transparent. Die Autoren schreiben selbst, dass nicht alle Teilaspekte aufgelistet werden konnten und dass sich die Noten „nicht nur aus den aufgeführten Punkten“" ergäben. ${ }^{13}$

(7) Die einzelnen Indikatoren der „Volksentscheid-Rankings“ werden als „hoch“ beziehungsweise „gering“ gewichtet, ohne aber diese Gewichte näher zu spezifizieren. ${ }^{14}$

Eine intersubjektive Überprüfung oder Rekonstruktion der einzelnen Bewertungen ist aufgrund der beschriebenen Vorgehensweise bei der Indexbildung nicht möglich. Aus wissenschaftlicher Sicht stellt sich neben dem Mangel an Transparenz daher auch die Frage nach der Validität der „Volksentscheid-Rankings“.

Aufbauend auf den Indizes von Christian Moser ${ }^{15}$ und Alois Stutzer ${ }^{16}$, die die Zugangshürden zu direktdemokratischen Verfahren in den schweizerischen Kantonen erfasst haben, und dem beschriebenen Ansatz von Mehr Demokratie e.V., soll hier ein neuer Messvorschlag für die Offenheit der Volksgesetzgebung in den Bundesländern vorgestellt werden. Dabei werden die Verfahrensaspekte in zwei additiven Indizes abgebildet: Ein erster Index misst die Offenheit der Strukturen auf dem Stand von Ende 2005. In einem zweiten Index wird die Offenheit für den Zeitraum zwischen 1990 und 2005 erfasst, wobei etwaige Änderungen der Vorschriften beachtet werden. Die genannten Kritikpunkte am Vorgehen der

9 Ralph Kampwirth, a.a.O., S. 13; Frank Rehmet / Karin Flothmann / Tim Weber, a.a.O., S. 13.

10 Ralph Kampwirth, a.a.O., S. 19; Frank Rehmet / Karin Flothmann / Tim Weber, a.a.O., S. 19 sowie Tabellen 7 und 10 dieses Beitrags.

11 Ralph Kampwirth, a.a.O., S. 20; Frank Rehmet / Karin Flothmann / Tim Weber, a.a.O., S. 20.

12 Ralph Kampwirth, a.a.O., S. 14; Frank Rehmet / Karin Flothmann / Tim Weber, a.a.O., S. 14.

13 Ralph Kampwirth, a.a.O., S. 27; Frank Rehmet / Karin Flothmann / Tim Weber, a.a.O., S. 27.

14 Ralph Kampwirth, a.a.O., S. 13; Frank Rehmet / Karin Flothmann / Tim Weber, a.a.O., S. 13.

15 Christian Moser, Institutionen und Verfahren der Rechtsetzung in den Kantonen, Bern 1985.

16 Alois Stutzer, Demokratieindizes für die Kantone der Schweiz, in: University of Zurich, Institute for Empirical Research in Economics (1999), Working Paper No. 23. 
Autoren der „Volksentscheid-Rankings“ wurden dabei berücksichtigt:

(1) Es wurde versucht, sowohl die Nachvollziehbarkeit als auch die Vollständigkeit der Messung deutlich zu erhöhen. Dazu wurden außer der einschlägigen Literatur auch die entsprechenden Rechtsquellen (Verfassungen, Ausführungsgesetze, Verordnungen) durchleuchtet.

(2) Auf eine Untersuchung der Rechtsprechungspraxis wird verzichtet, da diese nicht als unmittelbarer Teil der institutionellen Strukturen gelten kann. Gleiches gilt für die Intensität der Reformdiskussion.

(3) Die Betrachtung thematischer Beschränkungen erweist sich aus unserer Sicht als wenig ergiebig, da das Finanztabu nach wie vor ein recht einheitlich verbreitetes Merkmal darstellt.

(4) Alle Einzelindikatoren gehen ungewichtet in den Index ein, da eine exakte Quantifizierung der Bedeutung nicht zu realisieren ist.

(5) Die Vergabe der einzelnen Werte wird im Detail ausgeführt, um ein Maximum an Transparenz zu erreichen.

(6) Zur Sicherstellung der Validität der Messung wurden für alle Indikatoren bis zu fünf Kategorien entwickelt, um so die institutionellen Strukturen möglichst wirklichkeitsgetreu abzubilden.

\section{Organisation und Ablauf des Volksgesetzgebungsverfahrens}

Im Folgenden soll durch die Bildung zweier Indizes die Frage beantwortet werden, wie einfach oder schwierig es in den einzelnen Bundesländern ist, ein Volksgesetzgebungsverfahren durchzuführen. Dabei liefert der erste Index eine Momentaufnahme der Volksgesetzgebung zum Ende des Jahres 2005, der zweite Index deckt den Zeitraum zwischen 1990 und 2005 ab. Je höher diese Indexwerte sind, desto offener ist das direktdemokratische System des Bundeslandes und desto leichter ist es, Volksgesetzgebungsverfahren durchzuführen.

Die Bildung der beiden Indizes erfolgt nach dem gleichen Muster: Das Gesamtverfahren wird zunächst in drei Phasen untergliedert, denen je fünf einzelne Verfahrenselemente (Indikatoren) zugeordnet werden. Diesen Indikatoren werden nach dem in Tabelle 1 abgebildeten Schema Werte auf dem Kontinuum zwischen „0“ (geschlossen) und „1“ (offen) zugewiesen, wobei der Wert „1“ einen theoretischen Maximalwert darstellt, der jedoch nicht notwendigerweise erreicht werden muss. Für die fünf Indikatorenwerte jeder Phase wird ein Durchschnittswert gebildet. Der Wert des Gesamtindexes ergibt sich als Durchschnittswert der drei Phasenwerte. ${ }^{17}$

Die den beiden Offenheitsindizes zugrunde liegenden Daten entstammen den Verfassungen, Ausführungsgesetzen und -verordnungen der Bundesländer. Für den zweiten Index, der den Zeitraum zwischen 1990 und 2005 erfasst, wird das Jahr der Verabschiedung des Ausführungsgesetzes als erstes Geltungsjahr der Volksgesetzgebung angenommen. In

17 Formeln zur Berechnung der Offenheitsindexwerte für das Bundesland X zum Zeitpunkt t:

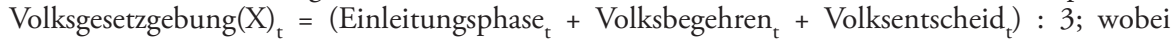
Einleitungsphase $\left(\mathrm{X}_{\mathrm{t}}=\left(\right.\right.$ Quorum $_{\mathrm{t}}+$ Frist $_{\mathrm{t}}+$ Modell $_{\mathrm{t}}+$ Bekanntmachung $_{\mathrm{t}}+$ Gericht $\left._{\mathrm{t}}\right): 5$; Volksbe- $_{\mathrm{t}}$ gehren $(\mathrm{X})_{\mathrm{t}}=\left(\right.$ Quorum $_{\mathrm{t}}+$ Frist $_{\mathrm{t}}+$ Sammlung $_{\mathrm{t}}+$ Bekanntmachung $_{\mathrm{t}}+$ Kostenerstattung $\left._{\mathrm{t}}\right): 5$; Volks- $_{\mathrm{t}}$

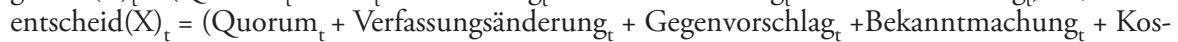
tenerstattung $\left._{\mathrm{t}}\right): 5$. 
diesen Gesetzen werden die meist relativ allgemein gehaltenen Vorschriften aus den Landesverfassungen konkretisiert und damit erst praktisch nutzbar gemacht. ${ }^{18}$ Dadurch ergeben sich unterschiedliche Beobachtungszeiträume: Die Hamburger Regelungen etwa stammen aus dem Jahr 1996, so dass für die Hansestadt insgesamt ein zeitlicher Rahmen von zehn Jahren veranschlagt wird. Für alle Länder, die das Instrument schon vor 1990 einführten, wird hingegen mit 16 Jahren gerechnet. Wurden in einem Bundesland die Verfahrensregeln im Untersuchungszeitraum geändert, so werden die alte und die neue Regelung getrennt bewertet. Diese Werte fließen dann entsprechend ihrer Geltungsdauer in Jahren in den Index ein. ${ }^{19}$

Die Volksgesetzgebung folgt in allen Bundesländern einem ähnlichen Ablaufschema: Vorgesehen sind grundsätzlich eine Einleitungsphase, ein Volksbegehren und ein Volksentscheid. In der Einleitungsphase müssen die Initiatoren einen Antrag einreichen, für den eine festgelegte Anzahl an Unterschriften nachzuweisen ist. Anschließend folgt das Volksbegehren, für das eine um ein Vielfaches höhere Zahl an Unterschriften als in der Einleitungsphase gesammelt werden muss. Bei Erreichen des entsprechenden Quorums kommt es zum Volksentscheid und damit zur verbindlichen Abstimmung über den fraglichen Gegenstand. Der Landtag kann dabei, außer in Bremen, einen Gegenvorschlag formulieren, den er dem Volk als Alternative im Volksentscheid unterbreitet.

\subsection{Einleitungsphase}

In der Einleitungsphase unterscheiden sich die Länder im Verhältnis zwischen der direkten und der parlamentarischen Gesetzgebung (Indikator 1.a): Entweder befasst sich der Landtag erst unmittelbar vor dem Volksentscheid mit der Vorlage der Initiatoren (konventionelles Modell), oder er entscheidet bereits vor dem Volksbegehren über das Anliegen (antizipierendes Modell), wodurch von Beginn an eine Kompromissbildung zwischen den Initiatoren und der regierenden Mehrheit im Landtag gefördert wird. ${ }^{20}$

Die beiden Modelle können nun in je eine Grundform und eine Variante differenziert werden. Bei der Variante werden den Initiatoren gegenüber der Grundform jeweils Mitbestimmungsmöglichkeiten über den Prozessablauf eingeräumt (vgl. Tabelle 2).

In der Grundform des konventionellen Modells durchläuft der Antrag das klassische Zulassungsverfahren: Es wird lediglich auf materielle und formelle Mängel hin geprüft. Bei

18 So regelt das Ausführungsgesetz für Volksgesetzgebungsverfahren gewöhnlich den Adressaten des Antrags auf Zulassung, die Beschaffenheit der Unterschriftenlisten im Volksbegehren oder inwieweit das Landtagswahlrecht auf den Volksentscheid anzuwenden ist.

19 In Hamburg wurde beispielsweise im Jahr 2001 das Quorum in der Einleitungsphase (Indikator 2b) von 1,6 auf 0,8 Prozent der Stimmberechtigten abgesenkt, was einer Veränderung des Indikatorenwertes von 0 auf 0,25 entspricht. Bei einer Gesamtgeltungsdauer von zehn Jahren waren beide Regelungen je fünf Jahre lang in Kraft. Die beiden Teilwerte fließen nun entsprechend ihrer gleichen Geltungsdauer je zur Hälfte in den Indikatorwert für den gesamten Untersuchungszeitraum ein, der somit einen Wert von 0,125 annimmt.

20 Vgl. auch Raphael Magin / Christina Eder, a.a.O. Schaal spricht in diesem Zusammenhang von einem offenen und einem geschlossenen Modell: Diana Schaal, Das Verfahren zur Herbeiführung eines Volksentscheids als Filter am Beispiel eines Drei-Länder-Vergleichs, in: Theo Schiller / Volker Mittendorf(Hrsg.), a.a.O., S. $153-164$. 


\begin{tabular}{|c|c|c|c|c|c|}
\hline \multicolumn{6}{|c|}{ Tabelle 1: Kodierung des Offenheitsindexes zur Messung von Volksgesetzgebungsverfahren } \\
\hline Verfahrensphase & geschlossen & $\ldots$ & \begin{tabular}{l|l}
$\ldots$ & \\
\end{tabular} & $\ldots$ & offen \\
\hline x) Indikator & 0 & 0,25 & 0,5 & 0,75 & 1 \\
\hline \multicolumn{6}{|l|}{ 1. Einleitungsphase } \\
\hline a) Modell & $\begin{array}{l}\text { konventionel- } \\
\text { les Modell - } \\
\text { Grundform } \\
\end{array}$ & $\begin{array}{l}\text { konventionel- } \\
\text { les Modell } \\
\text { - Variante } \\
\end{array}$ & $\begin{array}{l}\text { antizipierendes } \\
\text { Modell - Grund- } \\
\text { form }\end{array}$ & $\begin{array}{l}\text { antizipierendes } \\
\text { Modell } \\
\text { - Variante } \\
\end{array}$ & $\begin{array}{l}\text { Mecklenburg- } \\
\text { Vorpommern }\end{array}$ \\
\hline $\begin{array}{l}\text { b) Quorum (in Prozent } \\
\text { der Stimmberechtigten) }\end{array}$ & $>1,01 \%$ & 0,76 bis $1,0 \%$ & 0,51 bis $0,75 \%$ & 0,26 bis $0,5 \%$ & 0 bis $0,25 \%$ \\
\hline c) Frist (in Tagen) & 0 bis 120 & 121 bis 240 & 241 bis 360 & $>361$ & keine \\
\hline $\begin{array}{l}\text { d) Bekanntmachung } \\
\text { (stets amtlich) }\end{array}$ & $\begin{array}{l}\text { keine weitere } \\
\text { Bekannt- } \\
\text { machung }\end{array}$ & $\begin{array}{l}\text { Bekannt- } \\
\text { machung der } \\
\text { Ablehnungs- } \\
\text { entscheidung }\end{array}$ & $\begin{array}{l}\text { Bekanntmachung } \\
\text { der Ablehnungs- } \\
\text { und Zulassungs- } \\
\text { entscheidung }\end{array}$ & $\begin{array}{l}\text { Bekanntmachung } \\
\text { der Ablehnungs- } \\
\text { und Zulassungsent- } \\
\text { scheidung sowie } \\
\text { der Begründung }\end{array}$ & $\begin{array}{l}\text { Bekanntmachung } \\
\text { des beabsichtigten } \\
\text { Verfahrens VOR } \\
\text { Beginn der Unter- } \\
\text { schriftensammlung }\end{array}$ \\
\hline e) gerichtliche Prüfung & $\begin{array}{l}\text { nur auf } \\
\text { Antrag }\end{array}$ & $\ldots$ & $\begin{array}{l}\text { auf Antrag einer } \\
\text { Landtagsminder- } \\
\quad \text { heit }\end{array}$ & $\ldots$ & $\begin{array}{l}\text { automatisch bei } \\
\text { Ablehnung }\end{array}$ \\
\hline Einleitung GESAMT & 0,00 & $\ldots$ & $\ldots$ & $\ldots$ & 1,00 \\
\hline \multicolumn{6}{|l|}{ 2. Volksbegehren } \\
\hline $\begin{array}{l}\text { a) Quorum (in Prozent } \\
\text { der Stimmberechtigten) }\end{array}$ & $>16 \%$ & 12,1 bis $16 \%$ & 8,1 bis $12 \%$ & 4,1 bis $8 \%$ & 0 bis $4 \%$ \\
\hline b) Frist (in Tagen) & 0 bis 30 & 31 bis 60 & 61 bis 90 & $>91$ & keine \\
\hline c) Sammlungsmodus & Amt & $\ldots$ & $\ldots$ & $\ldots$ & frei \\
\hline d) Bekanntmachung & $\begin{array}{l}\text { Formalia und } \\
\text { Gegenstand } \\
\text { (amtlich) }\end{array}$ & \multicolumn{4}{|c|}{$\begin{array}{l}\text { Begründung, benötigte Stimmen, nichtamtliche Veröffentlichung, } \\
\text { Vorlage im Wortlaut } \\
\text { (je }+0,25 \text { Punkte) }\end{array}$} \\
\hline e) Kostenerstattung & keine & $\begin{array}{l}\text { Listen (erfolgs- } \\
\text { bedingt) }\end{array}$ & Listen & $\begin{array}{c}\leq 0,10 € \\
\text { pro Stimme }\end{array}$ & $\begin{array}{l}\geq 0,11 € \\
\text { pro Stimme }\end{array}$ \\
\hline Volksbegehren gesamt & 0,00 & $\ldots$ & $\ldots$ & $\ldots$ & 1,00 \\
\hline \multicolumn{6}{|l|}{ 3. Volksentscheid } \\
\hline $\begin{array}{l}\text { a) Quorum für einfache } \\
\text { Gesetze (in Prozent der } \\
\text { Stimmberechtigten) }\end{array}$ & $\begin{array}{l}>33,3 \% \mathrm{Zu}- \\
\text { stimmungs- } \\
\text { quorum / } \\
50 \% \\
\text { Beteiligungs- } \\
\text { quorum }\end{array}$ & $\begin{array}{c}16,7 \text { bis } 33,3 \\
\% \text { Zustim- } \\
\text { mungs- } \\
\text { quorum }\end{array}$ & $\begin{array}{c}0 \text { bis } 16,6 \% \\
\text { Zustimmungs- } \\
\text { quorum }\end{array}$ & $\begin{array}{c}25 \% \text { Beteiligungs- } \\
\text { quorum }\end{array}$ & $\begin{array}{l}\text { einfache } \\
\text { Mehrheit }\end{array}$ \\
\hline $\begin{array}{l}\text { b) Quorum für Verfas- } \\
\text { sungsänderungen (in } \\
\text { Prozent der Stimm- } \\
\text { berechtigten) }\end{array}$ & $\begin{array}{c}>33,3 \% \mathrm{Zu}- \\
\text { stimmungs- } \\
\text { quorum / } \\
50 \% \\
\text { Beteiligungs- } \\
\text { quorum / } \\
\text { nicht möglich }\end{array}$ & $\begin{array}{c}16,7 \text { bis } 33,3 \\
\% \text { Zustim- } \\
\text { mungsquorum }\end{array}$ & $\begin{array}{c}0 \text { bis } 16,6 \% \\
\text { Zustimmungs- } \\
\text { quorum }\end{array}$ & $\begin{array}{c}25 \% \text { Beteiligungs- } \\
\text { quorum }\end{array}$ & $\begin{array}{l}\text { einfache } \\
\text { Mehrheit }\end{array}$ \\
\hline c) Gegenvorschlag & nein & $\ldots$ & $\ldots$ & $\ldots$ & ja \\
\hline d) Bekanntmachung & $\begin{array}{l}\text { Formalia und } \\
\text { Gegenstand } \\
\text { (amtlich) }\end{array}$ & $\begin{array}{l}\text { Vorlage im } \\
\text { Wortlaut } \\
\text { (amtlich) }\end{array}$ & $\begin{array}{l}\text { Begründung der } \\
\text { Antragsteller und } \\
\text { Position der Regie- } \\
\text { rung / des Landtags } \\
\text { (amtlich) }\end{array}$ & $\begin{array}{c}\text { Broschüre mit Vor- } \\
\text { lage im Wortlaut }\end{array}$ & $\begin{array}{l}\text { Broschüre mit } \\
\text { Begründung der } \\
\text { Antragsteller und } \\
\text { Position der Regie- } \\
\text { rung / des Landtags }\end{array}$ \\
\hline & \multicolumn{5}{|c|}{ weitere Veröffentlichung je $+0,25$ Punkte } \\
\hline e) Kostenerstattung & keine & erfolgsbedingt & $\begin{array}{c}<0,10 € \\
\text { pro Stimme }\end{array}$ & $\begin{array}{l}0,10 \text { bis } 0,20 € \\
\text { pro Stimme }\end{array}$ & $\begin{array}{l}\geq 0,21 € \\
\text { pro Stimme }\end{array}$ \\
\hline Volksentscheid gesamt & 0,00 & $\ldots$ & $\ldots$ & $\ldots$ & 1,00 \\
\hline $\begin{array}{l}\text { Volksgesetzgebung } \\
\text { insgesamt }\end{array}$ & 0,00 & $\cdots$ & $\cdots$ & $\cdots$ & 1,00 \\
\hline
\end{tabular}


Tabelle 2: Gegenüberstellung konventionelles und antizipierendes Modell (Indikator 1.a)

\begin{tabular}{|c|c|c|c|c|}
\hline & \multicolumn{2}{|c|}{ konventionelles Modell } & \multicolumn{2}{c|}{ antizipierendes Modell } \\
\cline { 2 - 5 } & Grundform & Variante & Grundform & Variante \\
\hline eigenständige VI & & möglich & & \\
\hline Parlamentsphase & & JA & & \\
\hline Einleitungsphase & $\begin{array}{c}\text { klassische } \\
\text { Zulassung }\end{array}$ & $\begin{array}{c}\text { klassische } \\
\text { Zulassung }\end{array}$ & VI & $\begin{array}{c}\text { VI / klass. } \\
\text { Zulassung }\end{array}$ \\
\hline Parlamentsphase & nein & nein & JA & JA / nein \\
\hline \multirow{2}{*}{ Länder (Stand 2005) } & BW, BY, & BE, HB, NI, & BB, HH, & RP, ST, (MV) \\
\hline Indexwert & HE, SL & NW, SH & 0,5 & 0,75 (MV = 1) \\
\hline
\end{tabular}

$\mathrm{VI}=$ Volksinitiative

Die Bundesländer werden im gesamten Beitrag wie folgt abgekürzt: BW = Baden-Württemberg, BY = Bayern, $\mathrm{BE}=$ Berlin, $\mathrm{BB}=$ Brandenburg, $\mathrm{HB}=$ Bremen, $\mathrm{HH}=$ Hamburg, $\mathrm{HE}=$ Hessen, $\mathrm{MV}=$ Mecklenburg-Vorpommern, NI = Niedersachsen, NW = Nordrhein-Westfalen, RP $=$ Rheinland-Pfalz, $\mathrm{SL}=$ Saarland, SN = Sachsen, ST = Sachsen-Anhalt, $\mathrm{SH}=$ Schleswig-Holstein, $\mathrm{TH}=$ Thüringen.

Quelle: Eigene Zusammenstellung.

der Variante dieses Modells ist zusätzlich eine fakultative Volksinitiative ${ }^{21}$ möglich. Wird eine solche Volksinitiative lanciert und lehnt der Landtag diese ab, müssen die Initiatoren erneut einen Zulassungsantrag stellen, um ihr Vorhaben auf dem Weg der Volksgesetzgebung weiter zu verfolgen. Die Volksinitiative wird demnach außerhalb der drei üblichen Verfahrensphasen Einleitung, Volksbegehren und Volksentscheid durchgeführt.

Das antizipierende Modell sieht in seiner Grundform die Volksinitiative regulär als erste Verfahrensstufe vor: Der Antrag der Initiatoren muss direkt vom Landtag behandelt werden. Lehnt dieser ab, kann ein Volksbegehren unmittelbar eingeleitet werden - ein gesondertes Zulassungsverfahren ist nicht mehr vonnöten. Bei der Variante des antizipierenden Modells kann in der Einleitungsphase zwischen der Volksinitiative und dem klassischen Zulassungsverfahren gewählt werden. Wenn sich die Initiatoren für das Zulassungsverfahren entscheiden, werden deutlich weniger Unterschriften im ersten Verfahrensschritt benötigt; allerdings muss zunächst die Stufe des Volksbegehrens gemeistert werden, bevor sich der Landtag mit der Vorlage beschäftigt. Wählen die Initiatoren hingegen die Volksinitiative als ersten Verfahrensschritt, so ist zwar eine erheblich größere Zahl an Unterschriften zu sammeln, der Landtag wird auf diese Weise jedoch unmittelbar mit dem Anliegen befasst.

Mecklenburg-Vorpommern ist nicht eindeutig einzuordnen, denn die Einleitungsphase wurde hier in das Volksbegehren integriert: Der Zulassungsantrag wird gemeinsam mit den Unterschriften für das Volksbegehren eingereicht; eine gesonderte Einleitungsphase ist

21 Unter einer Volksinitiative wird im vorliegenden Beitrag ein Antrag aus der Mitte der Stimmberechtigten verstanden, der wie auch die Volksgesetzgebung die Verabschiedung eines Gesetzes zum Ziel hat. Im Unterschied zur Volksgesetzgebung befasst die Volksinitiative jedoch lediglich den Landtag mit einer bestimmten Materie. Dem Landtag steht es sodann frei, ein Gesetz über den eingebrachten Gegenstand zu beschließen oder den Antrag ohne weitere Konsequenzen abzulehnen. Die Initiative dient in einigen Bundesländern als erste Stufe des Volksgesetzgebungsverfahrens - siehe dazu auch die unten folgende Beschreibung der Einleitungsphase. 
nicht vorgesehen. ${ }^{22}$ Soll der Landtag unmittelbar mit einem Anliegen befasst werden, steht den Bürgern zusätzlich zu diesem verkürzten Verfahren eine eigenständige Volksinitiative zur Verfügung.

Für den Offenheitsindex wird dem konventionellen Modell in der Grundform der niedrigste Wert zugeordnet, da hier von den Initiatoren die höchsten Hürden überwunden werden müssen: Im Vergleich zum antizipierenden Modell müssen ein erfolgreicher Antrag und ein erfolgreiches Volksbegehren vorliegen, bevor sich der Landtag das erste Mal mit dem Anliegen befasst und sich somit eine erste Möglichkeit der Verwirklichung des Anliegens bietet. Das in Mecklenburg-Vorpommern geltende Modell der Volksgesetzgebung sieht gar keine Einleitung vor, wenn auch eine fakultative Volksinitiative möglich ist. Aufgrund der fehlenden ersten Phase und des somit reduzierten Aufwandes für die Initiatoren wird dem Volksgesetzgebungsverfahren nach diesem Modell der höchste Offenheitsgrad zugeschrieben. Die übrigen Modelle gliedern sich wie aus Tabelle 2 ersichtlich zwischen diesen zwei Extrempunkten ein.

Verglichen mit dem in der zweiten Phase folgenden Volksbegehren sind die Anzahl der zu sammelnden Unterschriften in der Einleitungsphase gering und die Sammelfristen lang (vgl. Tabelle 3). Bei klassischen Zulassungsverfahren variieren diese Einleitungsquoren (Indikator 1.b) zwischen 0,02 Prozent der Stimmberechtigten in Nordrhein-Westfalen und 3,0 Prozent in Hessen, der Durchschnitt beträgt 0,65 Prozent. ${ }^{23}$ Die Sammelfristen (Indikator 1.c) betragen zwischen zwei Monaten und zwei Jahren, in einigen Ländern unterliegen sie keinerlei zeitlicher Beschränkung. Bei Volksinitiativen liegen die Einleitungsquoren höher als beim klassischen Zulassungsverfahren. Sie reichen von 0,5 Prozent in Nordrhein-Westfalen bis 3,7 Prozent in Berlin - bei einem Mittelwert von 1,4 Prozent. Die Sammelfristen sind tendenziell kürzer bemessen und beginnen in Nordrhein-Westfalen bei acht Wochen. Wie auch beim klassischen Zulassungsverfahren sind sie in einigen Ländern nicht zeitlich begrenzt.

Für die Indexkonstruktion wird davon ausgegangen, dass hohe Quoren und kurze Fristen auf geschlossene Systeme hindeuten, denn kleinen und unorganisierten Interessen wird so die Durchführung eines Verfahrens und damit die Erlangung öffentlicher Aufmerksamkeit für ihr Anliegen erschwert.

Die Art und Weise der öffentlichen Bekanntmachung von Volksgesetzgebungsverfahren (Indikator 1.d) wird in der Literatur häufig vernachlässigt. ${ }^{24}$ Dies überrascht angesichts der Rolle, die der Kommunikation im politischen Raum zugemessen wird. ${ }^{25}$ Da in den Bun-

22 Damit ist die Volksgesetzgebung in Mecklenburg-Vorpommern vergleichbar mit dem in den schweizerischen Kantonen üblichen Verfahren, in dem ebenfalls keine gesonderte Einleitungsphase vorgesehen ist.

23 In Rheinland-Pfalz kann nach $\$ 63$ Abs. 5 Landeswahlgesetz auf die Sammlung von Unterschriften verzichtet werden, falls eine im Landtag vertretene Partei den Zulassungsantrag beziehungsweise die Volksinitiative einreicht.

24 Die entsprechenden Regelungen werden, wenn überhaupt, lediglich in deskriptiver Weise wiedergegeben, vgl. zum Beispiel Hermann K. Heußner, Volksgesetzgebung in den USA und in Deutschland. Ein Vergleich der Normen, Funktionen, Probleme und Erfahrungen, Köln 1994; Gunther Jürgens, Direkte Demokratie in den Bundesländern. Gemeinsamkeiten - Unterschiede - Erfahrungen. Vorbildfunktion für den Bund?, Stuttgart 1993.

25 So betont Habermas, eine „legitime Rechtsetzung“ erfordere eine „Institutionalisierung von [...] Kommunikationsvoraussetzungen für eine diskursive Meinungs- und Willensbildung “; Jürgen Habermas, Faktizität und Geltung. Beiträge zur Diskurstheorie des Rechts und des demokratischen Rechtsstaats, Frankfurt am Main 1992. 


\begin{tabular}{|c|c|c|c|c|c|c|c|c|}
\hline \multicolumn{9}{|c|}{$\begin{array}{l}\text { Tabelle 3: Quoren und Sammelfristen für Einleitungen von Volksgesetzgebungsverfahren in den } \\
\text { Bundesländern (Indikatoren 1.b und 1.c; Stand 2005) }\end{array}$} \\
\hline \multirow{2}{*}{ ָ⿹气 } & \multicolumn{3}{|c|}{ Klassisches Zulassungsverfahren } & \multicolumn{3}{|c|}{ Volksinitiative } & \multirow{2}{*}{ 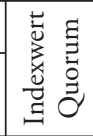 } & \multirow{2}{*}{ 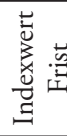 } \\
\hline & $\begin{array}{l}\text { Quorum } \\
\text { absolut }\end{array}$ & in $\%$ & Zulassungsfrist & $\begin{array}{c}\text { Quorum } \\
\text { absolut }\end{array}$ & in $\%$ & Zulassungsfrist & & \\
\hline $\mathrm{BW}$ & 10000 & 0,1 & keine Beschränkung & \multicolumn{3}{|c|}{ keine VI möglich } & 1 & 1 \\
\hline BY & & 0,3 & & \multicolumn{3}{|c|}{ keine VI möglich } & 0,75 & 0,75 \\
\hline $\mathrm{BE}$ & 25000 & 1 & 6 Monate & & 3,7 & $6 \mathrm{Mo}$ & 0,25 & 0,25 \\
\hline $\mathrm{BB}$ & \multicolumn{3}{|c|}{ VI statt Zulassungsverfahren } & 20000 & 0,9 & $1 \mathrm{Jahr}$ & 0,25 & 0,5 \\
\hline $\mathrm{HB}$ & 5000 & 1 & keine Beschränkung & & 2 & keine Beschränkung & 0,25 & 1 \\
\hline $\mathrm{HH}$ & \multicolumn{3}{|c|}{ VI statt Zulassungsverfahren } & & 0,8 & & 0,25 & 0,25 \\
\hline $\mathrm{HE}$ & 130000 & 3 & keine Beschränkung & \multicolumn{3}{|c|}{ keine VI möglich } & 0 & 1 \\
\hline MV & \multicolumn{3}{|c|}{ kein gesonderter Antrag erforderlich } & & 1,1 & keine Besch & 1 & 1 \\
\hline NI & 25000 & 0,4 & 6 Monate & 70000 & 1,2 & $1 \mathrm{Jahr}$ & 0,75 & 0,25 \\
\hline NW & 000 & 02 & keine Beschränkung & & 0,5 & & 1 & 1 \\
\hline $\mathrm{RP}$ & 20000 & 0,7 & & & 1 & & 0,25 & 0,75 \\
\hline SL & 5000 & 0,6 & 6 Monate & \multicolumn{3}{|c|}{ keine VI möglich } & 0,5 & 0,25 \\
\hline SN & \multicolumn{3}{|c|}{ VI statt Zulassungsverfahren } & & 1,1 & keine Besch & 0 & 1 \\
\hline ST & 8000 & 0,4 & keine Beschränkung & 30000 & 1,4 & keine Beschränkung & 0,25 & 1 \\
\hline $\mathrm{SH}$ & \multicolumn{3}{|c|}{ VI statt Zulassungsverfahren } & 20000 & 0,9 & $1 \mathrm{Jahr}$ & 0,25 & 0,5 \\
\hline TH & 5000 & 0,3 & 6 Wochen & & 2,6 & 6 Monate & 0,75 & 0 \\
\hline$\varnothing$ & & 0,65 & & & 1,4 & & & \\
\hline \multicolumn{9}{|c|}{$\begin{array}{l}\text { VI = Volksinitiative . } \\
\text { Die Quoren sind üblicherweise in absoluten Zahlen festgelegt. Als Prozentwert ist das Quorum im klassi- } \\
\text { schen Zulassungsverfahren nur in Hessen und in der Volksinitiative nur in Bremen und Nordrhein-West- } \\
\text { falen im Gesetz angegeben. Die Berechnung der Werte erfolgte aufgrund der Stimmberechtigtenzahlen } \\
\text { der jeweils letzten Landtagswahl (nach www.election.de, 19. Oktober 2006). } \\
\text { In Ländern, in denen die Einleitungsphase nach der Variante des konventionellen Modells angelegt ist } \\
\text { (BE, HB, NI, NW, TH), wurde das Quorum für die klassische Zulassung als Bewertungsgrundlage her- } \\
\text { angezogen, da die Volksinitiative hier eine zusätzliche, jedoch nicht notwendige Stufe im Volksgesetzge- } \\
\text { bungsverfahren darstellt. Ist die Einleitungsphase nach der Variante des antizipierenden Modells organi- } \\
\text { siert (RP, ST), wurde zwischen dem Quorum der klassischen Zulassung und dem der Volksinitiative ein } \\
\text { Mittelwert gebildet, denn eine erfolgreiche Volksinitiative ersetzt in diesem Fall das Zulassungsverfahren. } \\
\text { Quelle: Eigene Zusammenstellung. }\end{array}$} \\
\hline
\end{tabular}

desländern die direkte gegenüber der repräsentativen Demokratie immer noch eine eher außergewöhnliche und sehr unregelmäßig genutzte Beteiligungsform darstellt und daher einen weitaus geringeren Bekanntheitsgrad aufweist, kommt diesem Argument besondere Bedeutung zu (vgl. Tabelle 4).

Weiterhin verfügen gerade jene Interessen, die das Repräsentativsystem nur unzureichend berücksichtigt und die deswegen auf dem Wege der Volksgesetzgebung artikuliert werden, mitunter nicht über die notwendigen Ressourcen, um ihren Anliegen aus eigener Kraft ausreichend Gehör zu verschaffen. Eine von staatlicher Seite gewährleistete Bekanntmachung „von unten“ artikulierter Politikimpulse kann daher dazu beitragen, dass die kommunikativen Voraussetzungen für die Durchführung von Volksgesetzgebungsverfahren verbessert werden. Berücksichtigt man, dass die Mobilisierung möglichst vieler Bürger wie bei der direkten Demokratie angestrebt - eine breite Streuung politischer Informatio- 


\begin{tabular}{|l|l|c|}
\hline Tabelle 4: & Bekanntmachung von Volksgesetzgebungsverfahren in der Einleitungsphase in den \\
Bundesländern (Indikator 1.d, Stand 2005)
\end{tabular}

nen voraussetzt, so kann ein politisches System, dessen Volksgesetzgebungsregularien umfassende Bekanntmachungsvorgaben machen, als offen eingeordnet werden. ${ }^{26}$

Die Veröffentlichungsvorschriften unterscheiden sich deutlich voneinander: Die Bekanntmachung in der Einleitungsphase erfolgt, wenn vorgesehen, zwar stets in amtlichen Organen und meist nach Eingang des Antrages. Jedoch ist beispielsweise in Niedersachsen eine öffentliche Bekanntmachung vorgesehen, noch bevor mit der Sammlung der Unterschriften begonnen wird, welche dem Zulassungsantrag beizufügen sind. Auch dem Umfang nach unterscheiden sich die Vorschriften zum Teil erheblich.

In den meisten Bundesländern erfolgt eine gerichtliche Überprüfung (Indikator 1.e) der Entscheidung der Zulassungsstelle nur auf Antrag (Indexwert 0). Die Ausnahmen sind Bayern und Bremen, wo sie automatisch nach einer Ablehnung der Zulassung ausgelöst wird (Indexwert 1), sowie Berlin, Mecklenburg-Vorpommern und Schleswig-Holstein, wo eine Minderheit der Landtagsabgeordneten das Gericht anrufen kann (Indexwert 0,5). Es wird davon ausgegangen, dass eine Erleichterung der gerichtlichen Überprüfung zur Offenheit des Systems beiträgt, da auf diese Weise einer willkürlichen Ablehnung von Anträgen durch die regierende Mehrheit vorgebeugt wird.

\subsection{Volksbegehren}

Auf der Stufe des Volksbegehrens variiert nicht nur die Anzahl der zu sammelnden Unterschriften (Indikator 2.a) beträchtlich, sondern auch die Fristen (Indikator 2.b), innerhalb derer diese zusammengetragen werden müssen: Die Zahl der erforderlichen Unterschriften

26 Die Verbreitung von Informationen ist eine notwendige, wenn auch keine hinreichende Bedingung für die öffentliche Meinungsbildung, vgl. ebenda. 
reicht von 3,8 bis 20 Prozent der Stimmberechtigten; die Fristen betragen zwischen 14 Tagen und acht Monaten (vgl. Tabelle 5).

Ein weiterer Unterschied besteht im Modus der Unterschriftensammlung (Indikator 2.c): In den meisten Ländern werden die Eintragungslisten in Amtsräumen ausgelegt, im Gegensatz zur freien Unterschriftensammlung, die nur in sechs Ländern möglich ist. In Ländern mit hohen Quoren und kurzen Fristen wie in Baden-Württemberg, Hessen oder im Saarland ist durchweg das amtliche Verfahren vorgeschrieben. Die freie Sammlung deutet auf ein offenes, die Sammlung im Amt auf ein geschlossenes System hin. Dahinter steht die Annahme, dass die freie Sammlung beispielsweise in Einkaufsstraßen oder direkt an der Haustür besonders kleinen und unorganisierten Gruppen das Erreichen des vorgeschriebenen Quorums erleichtert. Große und organisierte Gruppen wie Gewerkschaften oder Ver-

\begin{tabular}{|c|c|c|c|c|c|c|}
\hline \multicolumn{7}{|c|}{$\begin{array}{l}\text { Tabelle 5: Verfahrensanforderungen (Quoren, Fristen und Sammlungsmodus) im Volksbegehren } \\
\text { in den Bundesländern (Indikatoren 2.a,b und c; Stand 2005) }\end{array}$} \\
\hline Land & $\begin{array}{c}\text { Quorum } \\
\text { (Stimmberechtigte } \\
\text { in Prozent) }\end{array}$ & $\begin{array}{l}\text { Indexwert } \\
\text { Quorum }\end{array}$ & $\begin{array}{c}\text { Eintragungs- } \\
\text { frist (in Tagen) }\end{array}$ & $\begin{array}{l}\text { Indexwert } \\
\text { Frist }\end{array}$ & $\begin{array}{l}\text { Sammlungs- } \\
\text { modus }\end{array}$ & $\begin{array}{l}\text { Indexwert } \\
\text { Sammlungs- } \\
\text { modus }\end{array}$ \\
\hline BW & 16,7 & 0 & 14 & 0 & Amt & 0 \\
\hline BY & 10 & 0,5 & 14 & 0 & Amt & 0 \\
\hline $\mathrm{BE}$ & 10 & 0,5 & 60 & 0,25 & Amt & 0 \\
\hline $\mathrm{BB}^{\mathrm{c}}$ & 3,8 & 1 & 120 & 0,75 & Amt & 0 \\
\hline $\mathrm{HB}^{\mathrm{a}}$ & $10 / 20$ & 0,5 & 90 & 0,5 & frei & 1 \\
\hline $\mathrm{HH}$ & 5 & 0,75 & 21 & 0 & Amt & 0 \\
\hline $\mathrm{HE}$ & 20 & 0 & 14 & 0 & Amt & 0 \\
\hline$M V^{c, d}$ & 10 & 0,5 & keine Frist & 1 & Amt/frei & 1 \\
\hline NI & 10 & 0,5 & 180 & 0,75 & frei & 1 \\
\hline NW & 8 & 0,5 & 56 & 0,25 & Amt & 0 \\
\hline $\mathrm{RP}^{\mathrm{c}}$ & 9,8 & 0,5 & 60 & 0,25 & Amt & 0 \\
\hline SL & 20 & 0 & 14 & 0 & Amt & 0 \\
\hline $\mathrm{SN}^{c}$ & 12,7 & 0,25 & 240 & 0,75 & frei & 1 \\
\hline ST & 11 & 0,5 & 180 & 0,75 & frei & 1 \\
\hline $\mathrm{SH}$ & 5 & 0,75 & 180 & 0,75 & Amt & 0 \\
\hline $\mathrm{TH}^{\mathrm{b}}$ & $08 / 10$ & 0,5 & $60 / 120$ & 0,75 & Amt/frei & 1 \\
\hline$\varnothing$ & 10,8 & & 85,2 & & & \\
\hline \multicolumn{7}{|c|}{$\begin{array}{l}\text { a Für Bremen bezieht sich der zweite Quorumswert auf verfassungsändernde Vorlagen. } \\
\text { b In Thüringen können die Initiatoren wählen, ob die Unterschriftensammlung frei oder in Amtsräumen } \\
\text { erfolgen soll. Der erste Wert bezieht sich jeweils auf die Vorgaben für die Sammlung in Amtsräumen, } \\
\text { der zweite Wert auf die freie Unterschriftensammlung. } \\
\text { c Die Quoren in Rheinland-Pfalz, Brandenburg, Mecklenburg-Vorpommern und Sachsen sind in der } \\
\text { jeweiligen Landesverfassung als Absolutwert festgelegt. Eigene Umrechnung aufgrund der Stimmbe- } \\
\text { rechtigtenzahlen bei der letzten Landtagswahl (nach www.election.de, 19. Oktober 2006). } \\
\text { d In Mecklenburg-Vorpommern ist die Unterschriftensammlung grundsätzlich frei. Eine Sammlung in } \\
\text { Amtsräumen kann jedoch für die Dauer von zwei Monaten zusätzlich beantragt werden, falls die Vor- } \\
\text { lage zuvor bereits als Volksinitiative eingebracht und vom Landtag zurückgewiesen wurde. } \\
\text { Quelle: Eigene Zusammenstellung. }\end{array}$} \\
\hline
\end{tabular}


bände sind vom Sammlungsmodus aufgrund ihrer breiten und leicht mobilisierbaren Mitgliederbasis weit weniger betroffen.

Die Regelungen zur Bekanntmachung im Volksbegehren (Indikator 2.d) werden analog zur Bekanntmachung in der Einleitungsphase analysiert. Das Minimum bilden dabei eine Veröffentlichung des Gegenstandes des Volksbegehrens sowie formaler Aspekte der Eintragung in die Unterschriftenlisten wie Auslegungsorte und deren Öffnungszeiten (bei amtlicher Eintragung), Beginn und Ende der Frist usw. Über diese Vorgaben hinausgehend sind mancherorts die Begründung der Initiatoren, der Wortlaut des begehrten Gesetzes sowie die benötigte Unterschriftenzahl für ein erfolgreiches Begehren bekanntzugeben. Bisweilen ist außer der amtlichen oder ortsüblichen Veröffentlichung auch für eine Bekanntmachung in nichtamtlichen Organen wie Zeitung oder Rundfunk zu sorgen (vgl. Tabelle 6).

Die einzelnen Inhalte und Modi der Bekanntmachung werden in den Bundesländern in unterschiedlicher Weise kombiniert. Daher lässt sich der Merkmalsraum, entgegen der sonstigen Vorgehensweise, nicht als Kontinuum abbilden, auf welchem die einzelnen Regelungen zwischen offen und geschlossen angeordnet werden können. Vielmehr trägt jeder zusätzliche Aspekt der Bekanntmachung, der in einem Bundesland vorgesehen ist, mit 0,25 Punkten zum Offenheitswert des Systems bei. Von einem offeneren System kann also ausgegangen werden, je umfangreicher die Bekanntmachung in einem Land ist.

Sowohl im Volksbegehren als auch im Volksentscheid kommt als weiteres Element die Möglichkeit der Kostenerstattung (Indikator 2.e) hinzu. Zunächst gilt generell, dass die Antragsteller die Kosten in der Einleitungsphase und im Volksbegehren selbst zu tragen haben. In den unten aufgeführten Bundesländern werden die Initiatoren direktdemokratischer Verfahren jedoch im Nachhinein für angefallene Kosten entschädigt. Dabei werden

\begin{tabular}{|l|l|c|}
\hline \multicolumn{3}{|l|}{ Tabelle 6: Bekanntmachung im Volksbegehren in den Bundesländern (Indikator 2.d, Stand 2005) } \\
\hline Land & Inhalt der Bekanntmachung & Indexwert \\
\hline BW & Formalia + Gegenstand, Wortlaut, Begründung & 0,5 \\
\hline BY & Formalia + Gegenstand & 0 \\
\hline BE & Formalia + Gegenstand, Vorlage im Wortlaut & 0,25 \\
\hline BB & Formalia + Gegenstand & 0 \\
\hline HB & Formalia + Gegenstand, Vorlage im Wortlaut, benötigte Stimmen & 0,5 \\
\hline HH & Formalia + Gegenstand, nichtamtliche Veröffentlichung & 0,25 \\
\hline HE & Formalia + Gegenstand, Vorlage im Wortlaut & 0,25 \\
\hline MV & Formalia + Gegenstand, Vorlage im Wortlaut & 0,25 \\
\hline NI & Formalia + Gegenstand & 0 \\
\hline NW & Formalia + Gegenstand & 0 \\
\hline RP & Formalia + Gegenstand, Vorlage im Wortlaut & 0,25 \\
\hline SL & Formalia + Gegenstand, Vorlage im Wortlaut & 0,25 \\
\hline SN & Formalia + Gegenstand, Vorlage im Wortlaut, Begründung & 0,5 \\
\hline ST & $\begin{array}{l}\text { Formalia + Gegenstand, Vorlage im Wortlaut, Begründung, benötigte } \\
\text { Stimmenanzahl }\end{array}$ & 0,75 \\
\hline SH & Formalia + Gegenstand, Vorlage im Wortlaut, Begründung & 0,5 \\
\hline TH & Formalia + Gegenstand, Vorlage im Wortlaut, Begründung & 0,5 \\
\hline Quelle: Eigene Zusammenstellung. & \\
\hline
\end{tabular}


entweder die Aufwendungen für die Durchführung des Verfahrens erstattet (Listenerstellung und Versand), oder es erfolgt eine Vergütung, die sich analog zur Wahlkampfkostenerstattung an der Resonanz des Verfahrens in der Bevölkerung orientiert (vgl. Tabelle 7). Es wird angenommen, dass eine umfangreiche Kostenerstattung für ein offenes System steht, da so gesellschaftliche Interessen unabhängig von ihrer materiellen Ausstattung eingebracht werden können.

\begin{tabular}{|l|l|c|}
\hline \multicolumn{1}{|c|}{ Tabelle 7: Kostenerstattung im Volksbegehren in den Bundesländern (Indikator 2.e, Stand 2005) } \\
\hline Land & \multicolumn{1}{|c|}{ Erstattungsregelung } & $\begin{array}{c}\text { Index- } \\
\text { wert }\end{array}$ \\
\hline NI & $\begin{array}{l}0,10 € \text { pro Unterschrift (bis zur Erfüllung des Quorums von zehn Prozent - circa } \\
60000 €)\end{array}$ & 0,75 \\
\hline NW & $\begin{array}{l}\text { Kosten für Listenherstellung und Versand } \\
\text { (nur bei erfolgreichem Volksentscheid) }\end{array}$ & 0,25 \\
\hline RP & Kosten für Listenherstellung und Versand (Erstattung auf Antrag) & 0,25 \\
\hline SN & $\begin{array}{l}0,51 € \text { pro 10 Unterschriften } \\
\text { (bis zur Erfüllung des Quorums von 450 000 Unterschriften - circa 23 000 €) }\end{array}$ & 0,75 \\
\hline ST & $\begin{array}{l}0,26 € \text { pro Unterschrift (bis zur Erfüllung des Quorums von elf Prozent - circa } \\
60000 €)\end{array}$ & 1 \\
\hline SH & Kosten für Versand der Listen & 0,5 \\
\hline \multicolumn{2}{|c|}{$\begin{array}{l}0,15 € \text { pro Unterschrift } \\
\text { (bis zur Erfüllung des Quorums von acht beziehungsweise zehn Prozent - circa }\end{array}$} & 1 \\
\hline \multicolumn{2}{|c|}{$23500 €$ beziehungsweise 30 000 $€$ ) } & \\
\hline $\begin{array}{l}\text { Nicht aufgeführt sind die Länder ohne Kostenerstattungsregelungen. Berechnung auf Basis der Stimm- } \\
\text { berechtigtenzahlen bei der letzten Landtagswahl (nach www.election.de, 19. Oktober 2006). In Baden- } \\
\text { Württemberg werden die Aufwendungen für das Zulassungsverfahren erstattet. } \\
\text { Quelle: Eigene Zusammenstellung. }\end{array}$ \\
\hline
\end{tabular}

\subsection{Volksentscheid}

Im Volksentscheid differieren die Quoren von Land zu Land (vgl. Tabelle 8). Die Kodierung erfolgt dabei nach derselben Logik wie in der Einleitungsphase und im Volksbegehren: je niedriger das Quorum, desto offener das System. Weiterhin unterscheiden sich die Vorgaben auch danach, ob es sich um ein einfaches Gesetz (Indikator 3.a) oder um eine Verfassungsänderung (Indikator 3.b) handelt.

Bis auf Bremen erlauben alle Landesverfassungen dem jeweiligen Landtag die Ausarbeitung eines Gegenvorschlags (Indikator 3.c), der den Wählern zusammen mit dem ursprünglichen Vorhaben zur Abstimmung vorgelegt wird. Parlamentarische Gegenvorschläge werden oftmals als breite gesellschaftliche Kompromisse zwischen der Position der repräsentativdemokratischen Arena und den direktdemokratisch geäußerten Interessen formuliert. ${ }^{27}$ So können Widerstände in der Volksvertretung gegenüber den von den Bürgern

27 Vgl. zum Beispiel den Gegenvorschlag des Bayerischen Landtages beim Volksentscheid über das Abfallrecht: Otmar Jung, Der Volksentscheid über das Abfallrecht in Bayern am 17. Februar 1991, in: ZParl, 23. Jg. (1992), H. 1, S. 48 - 69. 


\begin{tabular}{|c|c|c|c|c|}
\hline Land & einfache Gesetze & Indexwert & verfassungsändernde Gesetze & Indexwert \\
\hline BW & $33 \%$ & 0,25 & $50 \%$ & 0 \\
\hline BY & einfache Mehrheit & 1 & $25 \%$ & 0,25 \\
\hline $\mathrm{BE}^{\mathrm{a}}$ & $33 \%$ & 0,25 & nicht vorgesehen & 0 \\
\hline $\mathrm{BB}$ & $25 \%$ & 0,25 & $\begin{array}{l}\text { 2/3-Mehrheit der Abstimmenden bei einer } \\
\text { Zustimmung von mindestens } 50 \%\end{array}$ & 0 \\
\hline $\mathrm{HB}^{\mathrm{d}}$ & $25 \%$ & 0,25 & $50 \%$ & 0 \\
\hline $\mathrm{HH}$ & $20 \%$ & 0,25 & $\begin{array}{l}\text { 2/3-Mehrheit der Abstimmenden bei einer } \\
\text { Zustimmung von mindestens } 50 \%\end{array}$ & 0 \\
\hline $\mathrm{HE}$ & einfache Mehrheit & 1 & nicht vorgesehen & 0 \\
\hline MV & $33 \%$ & 0,25 & $\begin{array}{l}\text { 2/3-Mehrheit der Abstimmenden bei einer } \\
\text { Zustimmung von mindestens } 50 \%\end{array}$ & 0 \\
\hline NI & $25 \%$ & 0,25 & $50 \%$ & 0 \\
\hline NWe & $15 \%$ & 0,5 & $\begin{array}{l}\text { 2/3-Mehrheit der Abstimmenden bei } \\
\text { mindestens } 50 \% \text { Beteiligung }\end{array}$ & 0 \\
\hline $\mathrm{RP}^{\mathrm{b}}$ & einfache Mehrheit & 0,75 & $50 \%$ & 0 \\
\hline SL & $50 \%$ & 0 & $\begin{array}{l}\text { kein Volksentscheid über die Verfassung } \\
\text { möglich, nur Volksbegehren }\end{array}$ & 0 \\
\hline SN & einfache Mehrheit & 1 & $50 \%$ & 0 \\
\hline $\mathrm{ST}^{\mathrm{c}}$ & $25 \%$ & 0,25 & $\begin{array}{l}\text { 2/3-Mehrheit der Abstimmenden bei einer } \\
\text { Zustimmung von mindestens } 50 \%\end{array}$ & 0 \\
\hline $\mathrm{SH}$ & $25 \%$ & 0,25 & $\begin{array}{l}\text { 2/3-Mehrheit der Abstimmenden bei einer } \\
\text { Zustimmung von mindestens } 50 \%\end{array}$ & 0 \\
\hline TH & $25 \%$ & 0,25 & $40 \%$ & 0 \\
\hline \multicolumn{5}{|c|}{$\begin{array}{l}\text { Zustimmungserfordernisse, soweit nicht anders angegeben. } \\
\text { a In Berlin entfällt bei mehr als } 50 \text { Prozent Beteiligung das Zustimmungsquorum für einfache Gesetze. } \\
\text { b In Rheinland-Pfalz ist bei einfachen Gesetzen zusätzlich zum Mehrheitserfordernis ein Beteiligungs- } \\
\text { quorum von } 25 \text { Prozent vorgesehen. } \\
\text { c In Sachsen-Anhalt entfällt das Quorum für einfachgesetzliche Abstimmungen, falls der Landtag einen } \\
\text { Gegenvorschlag eingereicht hat. } \\
\text { d In Bremen galt bis } 1994 \text { ein Beteiligungsquorum von } 50 \text { Prozent für einfache Gesetze. } \\
\text { e In Nordrhein-Westfalen waren bis } 2002 \text { Verfassungsänderungen auf dem Weg der Volksgesetzgebung } \\
\text { nicht vorgesehen. } \\
\text { Quelle: Eigene Zusammenstellung. }\end{array}$} \\
\hline
\end{tabular}

unmittelbar artikulierten Anliegen abgebaut werden. Wird im Volksentscheid der Gegenvorschlag statt der ursprünglichen Vorlage angenommen, ist dies aufgrund des Kompromisscharakters des Gegenvorschlags aus Sicht der Initiatoren immerhin noch als Teilerfolg zu werten. Es ist zudem davon auszugehen, dass ein Gegenvorschlag ein hohes Maß an Öffentlichkeit erzeugt. Insgesamt wird daher angenommen, dass die Möglichkeit eines Gegenvorschlags durch sein aktivierendes und vermittelndes Potential zur Offenheit des Volksgesetzgebungsverfahrens beiträgt.

Die Einordnung der Bekanntmachungsvorschriften im Volksentscheid (Indikator 3.d) folgt der bereits in der Einleitungsphase und im Volksbegehren angewandten Argumenta- 


\begin{tabular}{|l|l|c|}
\hline \multicolumn{2}{|l|}{ Tabelle 9: Bekanntmachung im Volksentscheid in den Bundesländern (Indikator 3.d, Stand 2005) } \\
\hline Land & Inhalt der Bekanntmachung & Indexwert \\
\hline BW & Formalia + Gegenstand, Broschüre mit Vorlage im Wortlaut & 0,75 \\
\hline BY & $\begin{array}{l}\text { Formalia + Gegenstand, Broschüre mit Vorlage im Wortlaut (bei zu langen } \\
\text { Gesetzen) }\end{array}$ & 0,75 \\
\hline BE & Formalia + Gegenstand, Vorlage im Wortlaut & 0,25 \\
\hline BB & Formalia + Gegenstand, Begründung und Gegenposition & 0,5 \\
\hline HB & Formalia + Gegenstand, Vorlage im Wortlaut & 0,25 \\
\hline HH & Formalia + Gegenstand, Broschüre mit Begründung und Gegenposition & 1 \\
\hline HE & Formalia + Gegenstand, Vorlage im Wortlaut & 0,25 \\
\hline MV & Formalia + Gegenstand, Begründung und Gegenposition & 0,5 \\
\hline NI & Formalia + Gegenstand, Begründung und Gegenposition & 0,5 \\
\hline NW & Formalia + Gegenstand, weitere nichtamtliche Veröffentlichung & 0,25 \\
\hline RP & Formalia + Gegenstand, Begründung und Gegenposition & 0,5 \\
\hline SL & Formalia + Gegenstand, Begründung und Gegenposition & 0,5 \\
\hline SN & Formalia + Gegenstand, Begründung und Gegenposition & 0,5 \\
\hline ST & Formalia + Gegenstand, Begründung und Gegenposition & 0,5 \\
\hline SH & Formalia + Gegenstand, Begründung und Gegenposition in geeigneter Form & 0,75 \\
\hline TH & Formalia + Gegenstand, Broschüre mit Begründung & 1 \\
\hline Quelle: Eigene Zusammenstellung. & \\
\hline
\end{tabular}

tion. Je umfassender die Inhalte der Bekanntmachung und je vielfältiger die Medien, desto offener das System. Dem Minimum entspricht hier, wie auch im Volksbegehren, die Veröffentlichung von Gegenstand und formalen Aspekten der Abstimmung (zum Beispiel Abstimmungsdatum und Stimmlokal). Weiterhin erhalten die Stimmberechtigten - in Analogie zur Wahlbenachrichtigung - in allen Bundesländern eine Abstimmungsbenachrichtigung, die ebenso rein formale Informationen enthält. Darüber hinausgehende Regelungen sehen die amtliche Veröffentlichung des Gesetzentwurfs im Wortlaut, die entsprechende Begründung der Antragsteller sowie die Darlegung der Position der Regierung oder des Landtages vor. In einigen Ländern werden den Stimmberechtigten Informationsbroschüren zugesandt, oder es hat parallel zur amtlichen Veröffentlichung eine zusätzliche Bekanntmachung in nichtamtlichen Organen wie Tageszeitungen oder Rundfunk zu erfolgen. Die Bestimmungen zur Bekanntmachung des Volksentscheids können gemäß ihres Inhalts und Umfangs auf einem Kontinuum zwischen geschlossen und offen angeordnet werden (vgl. Tabelle 9).

Die Kostenerstattung (Indikator 3.e) wird analog zum Volksbegehren bewertet, allerdings ändern sich die Erstattungsbeträge pro abgegebener Stimme (vgl. Tabelle 10). Insgesamt gibt es Kostenerstattungen nur in fünf Bundesländern. Die Kosten für die eigentliche Durchführung des Volksentscheids, das heißt für den Abstimmungsvorgang selbst, trägt stets das Land. 
Tabelle 10: Kostenerstattung im Volksentscheid in den Bundesländern (Indikator 3.e, Stand 2005)

\begin{tabular}{|l|l|c|}
\hline Land & Erstattungsregelung & Indexwert \\
\hline $\mathrm{HH}$ & $0,10 €$ pro Ja-Stimme (maximal $40000 €$ ) & 0,75 \\
\hline $\mathrm{SN}$ & $1,02 €$ pro 100 Ja-Stimmen & 0,5 \\
\hline $\mathrm{ST}$ & $0,26 €$ pro Ja-Stimme (bis 25 Prozent der Stimmberechtigten - circa 135000 €) & 1 \\
\hline $\mathrm{SH}$ & $0,28 €$ pro Ja-Stimme (maximal der Gesamtbetrag der Werbekosten) & 1 \\
\hline $\mathrm{TH}^{\mathrm{a}}$ & $\begin{array}{l}0,075 € \text { pro Ja-Stimme (nur bei erfolgreichem Volksentscheid, maximal die } \\
\text { Stimmenanzahl, die für die Annahme erforderlich war) }\end{array}$ & 0,25 \\
\hline $\begin{array}{l}\text { a In Thüringen ist bei einfachgesetzlichen Vorlagen im Volksentscheid ein Zustimmungsquorum von 25 } \\
\text { Prozent vorgesehen, was einem maximalen Erstattungsbetrag von circa 37 000 € entspräche. Bei verfas- } \\
\text { sungsändernden Vorlagen ist eine Zustimmung von 40 Prozent erforderlich, wodurch maximal circa } \\
60 \text { 000 € erstattet würden. } \\
\text { Quelle: Eigene Zusammenstellung. }\end{array}$ \\
\hline
\end{tabular}

\section{Die Offenheit der Volksgesetzgebung im Vergleich}

Addiert man die Werte der einzelnen Indikatoren wie zu Beginn von Abschnitt 2 beschrieben, kann man die Bundesländer anhand der Offenheitsindex-Werte von 2005 in eine Rangfolge bringen, der in Tabelle 11 zum Vergleich die Rankings von Ralph Kampwirth ${ }^{28}$ sowie Frank Rehmet, Karin Flothmann und Tim Weber ${ }^{29}$ hinzugefügt wurden.

Der hier vorgestellte Index offenbart ein erhebliches Maß an Varianz im Grad der Offenheit direktdemokratischer Verfahren: So ist beispielsweise der Wert von Sachsen-Anhalt, wo aufgrund der institutionellen Ausgestaltung die Durchführung eines Volksgesetzgebungsverfahrens am einfachsten ist, fast dreimal so hoch wie der des Saarlands, wo es vergleichsweise am schwierigsten ist, solche Verfahren zu initiieren. Die ersten vier Länder - Sachsen-Anhalt, Mecklenburg-Vorpommern, Sachsen und Schleswig-Holstein - bilden die Spitzengruppe. An zweiter Stelle folgt die Gruppe Thüringen, Niedersachsen, Bayern und Rheinland-Pfalz, anschließend Bremen, Brandenburg, Hamburg, Nordrhein-Westfalen und Baden-Württemberg. Das Schlusslicht bilden Berlin, Hessen und das Saarland. In den ersten beiden Gruppen finden sich - mit Ausnahme von Bayern und Rheinland-Pfalz - nur Länder, die die Volksgesetzgebung erst nach 1990 eingeführt haben. Umgekehrt findet man auf den letzten Plätzen - abgesehen von Berlin - nur Länder, in denen diese unmittelbaren Beteiligungsrechte schon vor der Wiedervereinigung vorhanden waren.

Vergleicht man den Offenheitsindex auf dem Stand von 2005 mit dem Ranking von Kampwirth, also dem ersten Volksentscheid-Ranking von Mehr Demokratie e.V., besteht der extremste Unterschied mit acht Plätzen bei Hamburg, danach folgen Bayern und Nordrhein-Westfalen mit je sechs. Eher gering sind die Unterschiede bei Berlin, Brandenburg, Niedersachsen, Sachsen und Thüringen. Gegenüber dem Ranking von Rehmet, Flothmann und Weber, dem zweiten von Mehr Demokratie e.V., nimmt Berlin mit zehn Plätzen Unterschied auf dem Offenheitsindex eine der hinteren Positionen ein, gefolgt von Mecklenburg-Vorpommern mit acht und Nordrhein-Westfalen mit 7,5 Plätzen Unterschied. Ähnli- 


\begin{tabular}{|c|c|c|c|c|c|c|}
\hline \multicolumn{7}{|c|}{$\begin{array}{l}\text { Tabelle 11: Der Offenheitsindex (Stand 2005) im Vergleich zu den Volksentscheid-Rankings } \\
\text { von Kampwirth und Rehmet / Flothmann / Weber }\end{array}$} \\
\hline \multirow{2}{*}{ Land } & \multicolumn{2}{|c|}{ Offenheitsindex (Stand 2005) } & \multicolumn{2}{|c|}{ Kampwirth } & \multicolumn{2}{|c|}{ Rehmet / Flothmann / Weber } \\
\hline & Indexwert & Rangplatz & Note & Rangplatz & Note & Rangplatz \\
\hline ST & 0,63 & 1 & 4,0 & 5 & 4,1 & 6 \\
\hline MV & 0,57 & 2 & 4,2 & 6 & 4,2 & 10 \\
\hline SN & 0,57 & 2 & 3,7 & 3 & 3,7 & 2 \\
\hline $\mathrm{SH}$ & 0,53 & 4 & 4,3 & 10 & 4 & 5 \\
\hline $\mathrm{TH}$ & 0,48 & 5 & 4,2 & 6 & 4,1 & 6 \\
\hline NI & 0,47 & 6 & 4,2 & 6 & 4,1 & 6 \\
\hline BY & 0,42 & 7 & 3,2 & 1 & 3,4 & 1 \\
\hline $\mathrm{RP}$ & 0,4 & 8 & 4,2 & 6 & 4,1 & 6 \\
\hline $\mathrm{HB}$ & 0,37 & 9 & 5,0 & 14 & 4,8 & 14 \\
\hline $\mathrm{BB}$ & 0,33 & 10 & 4,4 & 11 & 4,3 & 11 \\
\hline $\mathrm{HH}$ & 0,33 & 10 & 3,6 & 2 & 4,7 & 13 \\
\hline $\mathrm{NW}$ & 0,33 & 10 & 3,8 & 4 & 3,7 & 2 \\
\hline $\mathrm{BW}$ & 0,3 & 13 & 5,3 & 15 & 5,3 & 15 \\
\hline $\mathrm{BE}$ & 0,25 & 14 & 4,6 & 13 & 3,8 & 4 \\
\hline $\mathrm{HE}$ & 0,23 & 15 & 4,5 & 12 & 4,5 & 12 \\
\hline SL & 0,22 & 16 & 6,0 & 16 & 6,0 & 16 \\
\hline$\varnothing$ & 0,4 & & 4,33 & & 4,3 & \\
\hline \multicolumn{7}{|c|}{$\begin{array}{l}\text { Der Wertebereich beim Offenheitsindex (Stand 2005) reicht von } 0 \text { (geschlossen) bis } 1 \text { (offen), bei Kamp- } \\
\text { wirth und Rehmet / Flothmann / Weber von } 1 \text { (offen) bis } 6 \text { (geschlossen). } \\
\text { Bei den Werten vom ersten und zweiten Volksentscheid-Ranking (Kampwirth und Rehmet / Flothmann / } \\
\text { Weber) handelt es sich jeweils um die Teilnote und die Platzierung für die Landesebene; die dort betrach- } \\
\text { tete Kommunalebene wurde hier nicht berücksichtigt. } \\
\text { Quelle: Eigene Berechnung; Ralph Kampwirth, a.a.O.; Frank Rehmet / Karin Flothmann / Tim Weber, } \\
\text { a.a.O. }\end{array}$} \\
\hline
\end{tabular}

che Ränge zeigen sich für Niedersachsen, Rheinland-Pfalz, Sachsen und Schleswig-Holstein. Von allen drei Indizes wurden nur für das Saarland identische Rangplätze vergeben.

Prüft man die Zusammenhänge des Offenheitsindex und der Rankings von Mehr Demokratie e.V. mit Spearmans Rangkorrelation, so erhält man Koeffizientenwerte von 0,57 bei $\mathrm{p}=0,02$ (Kampwirth) beziehungsweise 0,47 bei $\mathrm{p}=0,07$ (Rehmet / Flothmann / Weber). ${ }^{30}$ Die positiven Vorzeichen besagen, dass höhere Werte auf dem Offenheitsindex tendenziell in jenen Ländern auftreten, die auch in den beiden Volksentscheid-Rankings „bessere“ Noten erhalten haben. Die Werte der Korrelationskoeffizienten lassen jedoch auf einen nur mittelstarken Zusammenhang schließen - eine Konsequenz der unterschiedlichen Grundlagen der drei Indizes:

30 Ein Koeffizient von +1 oder -1 deutet auf einen perfekten Zusammenhang, 0 dagegen auf keinen Zusammenhang hin. Zur Möglichkeit der Verwendung von Signifikanztests bei Vollerhebungen siehe Andreas Diekmann, Empirische Sozialforschung. Grundlagen, Methoden, Anwendungen, Reinbek 2005, S. 600, sowie Markus Freitag, Labor Schweiz: Vergleichende Wahlbeteiligungsforschung bei kantonalen Parlamentswahlen, in: Kölner Zeitschrift für Soziologie und Sozialpsychologie, 57. Jg. (2005), S. 667 - 690, Fußnote 13. 
Zwar handelt es sich bei allen drei um eine Momentaufnahme; der hier vorgestellte Offenheitsindex berücksichtigt jedoch den Stand der Regularien des Jahres 2005, bei Kampwirth ist es Herbst 2003, bei Rehmet / Flothmann / Weber Dezember 2007, so dass sich ein Teil der Unterschiede aus Änderungen im Regelwerk begründet. Aber auch die Vorgehensweise bei der Messung spielt eine maßgebliche Rolle. So wurden in den Offenheitsindex ausschließlich institutionelle Aspekte aufgenommen. Auf nicht rekonstruierbare Indikatoren wie die Rechtsprechungspraxis oder die Intensität der Reformdebatte in den einzelnen Bundesländern, die in die Volksentscheid-Rankings eingegangen sind, wurde bewusst verzichtet, da solche Größen nicht unmittelbar Teil des institutionellen Rahmens und zudem nur schwer operationalisierbar sind.

\section{Der Zusammenhang zwischen Offenheit und Nutzungsfrequenz der Volksgesetzgebung}

Der systematische Vergleich der institutionellen Rahmenbedingungen der Volksgesetzgebung in den Bundesländern stellt an sich bereits einen analytischen Mehrwert dar. Darüber hinaus ist eine Vielzahl an praktischen Anwendungsbereichen des Indexes denkbar. Eine dieser Möglichkeiten soll abschließend vorgestellt werden:

Die wohl populärste These im Zusammenhang mit der vergleichenden Analyse direktdemokratischer Institutionen besagt, dass die direkten Volksrechte in einem geschlossenen System eine geringere Nutzungsfrequenz als in einem offenen System aufweisen - eine Behauptung, die häufig auch für die deutschen Bundesländer geäußert wurde. ${ }^{31}$ Begründet wird dies durch den erhöhten Aufwand, den eine unmittelbare Interessensartikulation in einem geschlossenen System mit sich bringt. Iwan Barankay, Pascal Sciarini und Alexander Trechse ${ }^{32}$, Trechse ${ }^{33}$ sowie Adrian Vatter ${ }^{34}$ konnten für die schweizerischen Kantone keine Bestätigung dieser Hypothese finden, und auch für die US-amerikanischen Bundesstaaten ließ sich kein entsprechender Zusammenhang nachweisen. ${ }^{35}$ Moser $^{36}$ stellte für die Schweiz sogar einen umgekehrten Zusammenhang fest. In der deutschen Forschergemeinde hält sich die These trotz dieser Befunde - und das, obwohl bisher keinerlei systematische Untersuchungen der Bundesländer zu dieser Frage durchgeführt wurden. Mit unserem Offen-

31 Siehe Karsten Bugiel, Volkswille und repräsentative Entscheidung. Zulässigkeit und Zweckmäßigkeit von Volksabstimmungen nach dem Grundgesetz, Baden-Baden 1991; Otmar Jung, Grundsatzfragen der direkten Demokratie, in: Andreas Kost (Hrsg.), a.a.O., S. 312 - 366; Gunther Jürgens, a.a.O.; Ralph Kampwirth, Der ernüchterte Souverän. Bilanz und Perspektiven der direkten Demokratie in den 16 Bundesländern und auf Bundesebene, in: ZParl, 34. Jg. (2003), H. 4, S. 657 - 671; Christian Pestalozza, Der Popularvorbehalt. Direkte Demokratie in Deutschland, Berlin 1981; Frank Rehmet, a.a.O.; Bärbel Weixner, a.a.O.

32 Iwan Barankay / Pascal Sciarini / Alexander Trechsel, Institutional Openness and the Use of Referendum and Popular Initiatives. Evidence from the Swiss Cantons, in: Swiss Political Science Review, 9. Jg. (2003), S. $169-199$.

33 Alexander Trechsel, Feuerwerk Volksrechte. Die Volksabstimmungen in den schweizerischen Kantonen 1970-1996, Basel 2000.

34 Adrian Vatter, Kantonale Demokratien im Vergleich. Entstehungsgründe, Interaktionen und Wirkungen politischer Institutionen in den Schweizer Kantonen, Opladen 2002.

35 Vgl. California Commission on Campaign Financing, Democracy by Initiative. Shaping California's Fourth Branch of Government, Los Angeles 1992.

36 Christian Moser, a.a.O. 
heitsindex steht nun ein Instrument zur Verfügung, das zur Schließung dieser Forschungslücke beitragen kann. Im Zeitraum zwischen 1990 und 2005 wurden in den deutschen Bundesländern 114 Volksgesetzgebungsverfahren initiiert (vgl. Tabelle 12). ${ }^{37}$

\begin{tabular}{|l|c|c|c|c|c|}
\hline \multicolumn{2}{|c|}{ Tabelle 12: Der Offenheitsindex (1990 bis 2005) und die Verfahrensanzahl in den Bundesländern } \\
\hline Land & $\begin{array}{c}\text { Wert } \\
\text { Offenheitsindex } \\
\text { insgesamt }\end{array}$ & $\begin{array}{c}\text { Durchschnittliche } \\
\text { Anzahl Verfahren } \\
\text { pro Jahr seit } \\
\text { Einführung }\end{array}$ & $\begin{array}{c}\text { Wert } \\
\text { Teilindex } \\
\text { Einleitungsphase }\end{array}$ & $\begin{array}{c}\text { Wert } \\
\text { Teilindex } \\
\text { Volksbegehren }\end{array}$ & $\begin{array}{c}\text { Wert } \\
\text { Teilindex } \\
\text { Volksentscheid }\end{array}$ \\
\hline BW & 0,3 & 0,06 & 0,4 & 0,1 & 0,4 \\
\hline BY & 0,43 & 1,00 & 0,55 & 0,1 & 0,65 \\
\hline BE & 0,25 & 0,44 & 0,25 & 0,2 & 0,3 \\
\hline BB & 0,33 & 1,23 & 0,3 & 0,35 & 0,35 \\
\hline HB & 0,32 & 0,50 & 0,51 & 0,38 & 0,06 \\
\hline HH & 0,3 & 1,30 & 0,18 & 0,22 & 0,51 \\
\hline HE & 0,23 & 0,00 & 0,2 & 0,05 & 0,45 \\
\hline MV & 0,57 & 1,00 & 0,8 & 0,55 & 0,35 \\
\hline NI & 0,47 & 0,58 & 0,45 & 0,6 & 0,35 \\
\hline NW & 0,3 & 0,31 & 0,43 & 0,09 & 0,37 \\
\hline RP & 0,32 & 0,06 & 0,36 & 0,12 & 0,48 \\
\hline SL & 0,22 & 0,13 & 0,3 & 0,05 & 0,3 \\
\hline SN & 0,57 & 0,77 & 0,45 & 0,65 & 0,6 \\
\hline ST & 0,62 & 0,33 & 0,55 & 0,75 & 0,55 \\
\hline SH & 0,49 & 1,00 & 0,5 & 0,5 & 0,48 \\
\hline TH & 0,46 & 0,33 & 0,21 & 0,75 & 0,42 \\
\hline$\varnothing$ & 0,39 & 0,57 & 0,40 & 0,34 & 0,41 \\
\hline Det & & & & & 1 \\
\hline
\end{tabular}

Der Wertebereich des Offenheitsindexes (1990 bis 2005) reicht von 0 (geschlossen) bis 1 (offen). Die Informationen zur Anzahl der Verfahren pro Bundesland entstammen den Zulassungsstellen der Länder sowie Johannes Rux, Direkte Demokratie in Deutschland, Baden-Baden 2008. Die durchschnittliche Anzahl der Volksgesetzgebungsverfahren pro Jahr wird berechnet als absolute Anzahl Volksgesetzgebungsverfahren 1990 - 2005 dividiert durch die Anzahl der Jahre seit Verabschiedung der Ausführungsgesetze und -verordnungen im Untersuchungszeitraum. Die Werte der Länder, in denen dieses Instrument bereits vor der deutschen Einheit zur Verfügung stand, werden ab 1990 berechnet.

Der Datensatz, der die verschiedenen Merkmalsausprägungen der einzelnen Bundesländer für den Untersuchungszeitraum enthält, konnte hier aus Platzgründen nicht abgedruckt werden. Er ist bei den $\mathrm{Au}-$ toren auf Anfrage erhältlich.

Quellen: Eigene Berechnungen; Zulassungsstellen der Länder sowie Johannes Rux, a.a.O.

Eine Überprüfung des Zusammenhangs zwischen dem Grad der Offenheit und der Häufigkeit der Nutzung von Volksgesetzgebungsverfahren ergab einen Korrelationskoeffizienten

37 Diese Zahl ergibt sich aus den klassischen Volksgesetzgebungsverfahren und den eigenständigen Volksinitiativen, die aber, wie zu Beginn des Abschnitts 3 dargestellt, als Teil der Einleitungsphase dienen können. Die übrigen direktdemokratischen Verfahren wie die obligatorischen Verfassungsreferenden in Bayern und Hessen, Landtagsabberufungen sowie die Abstimmungen zur Verabschiedung der Landesverfassungen in den ostdeutschen Bundesländern bleiben unberücksichtigt. 
nach Pearson von $r=0,36$, was einem eher geringen Anteil erklärter Varianz von $R^{2}=13$ Prozent entspricht. Das Vorzeichen weist zwar in die angenommene Richtung, der Zusammenhang ist mit $\mathrm{p}=0,17$ allerdings nicht signifikant. ${ }^{38}$

Neben einem Einfluss der Offenheit des gesamten Verfahrens wäre auch ein besonderes Gewicht einzelner Verfahrensphasen denkbar. So könnten etwa die Hürden in der Einleitungsphase das entscheidende Hindernis darstellen und die nachfolgenden Phasen nur von nachgeordneter Relevanz sein. Auch diese Hypothese wurde mittels bivariater Korrelationen für alle drei Phasen separat getestet. Es zeigt sich, dass unter den drei Phasen zwar die Einleitungsphase den stärksten Zusammenhang mit der Häufigkeit der Volksgesetzgebung aufweist $(r=0,34)$, gefolgt von der Volksbegehrensphase $(r=0,22)$ und dem Volksentscheid ( $r$ $=0,2) .{ }^{39}$ Jedoch liegen die Werte der Koeffizienten für alle Teilindizes niedriger als für den Gesamtindex, so dass diesem die höchste Erklärungskraft zugeschrieben werden kann.

Um für einflussreiche Fälle zu kontrollieren, schlägt Ben Jann für Studien mit kleinen Fallzahlen eine Reihe von Verfahren zur Ermittlung von Ausreißern vor. ${ }^{40}$ Alle Ergebnisse dieser Diagnosen weisen auf Sachsen-Anhalt als Fall mit großem Einfluss auf das Gesamtergebnis hin. Hier wurden trotz eines eher offen ausgestalteten Volksgesetzgebungsverfahrens im Untersuchungszeitraum nur relativ wenige Verfahren angestrengt. Schließt man das Bundesland aus der Berechnung aus, steigt der Korrelationskoeffizient auf 0,5 an und wird mit p = 0,06 signifikant. Daraus lässt sich folgern, dass hohe Werte auf dem Offenheitsindex zu mehr Volksgesetzgebungsverfahren führen können. Der Anteil erklärter Varianz ist jedoch mit $\mathrm{R}^{2}=$ 25 Prozent immer noch gering, so dass dieses Resultat hier nur als Trend interpretiert wird.

Zusammenfassend bleibt festzuhalten, dass die gesetzlichen Vorschriften als alleinige Erklärung für die Varianz in der Nutzung der Volksgesetzgebung nicht ausreichen. Es ist anzunehmen, dass sozio-strukturelle, sozio-ökonomische oder auch kulturelle Faktoren, die in dieser Studie keine Beachtung finden, auf die Anwendungshäufigkeit wirken. Damit liegen nun für die deutschen Bundesländer ähnliche Befunde wie bereits für die schweizerischen Kantone $^{41}$ und die US-amerikanischen Bundesstaaten vor. ${ }^{42}$

\section{Fazit und Ausblick}

Der hier vorgestellte Index misst die Offenheit der Volksgesetzgebung in den deutschen Bundesländern seit der Wiedervereinigung aus komparativ-analytischer Perspektive. Dabei

38 Zur Möglichkeit der Verwendung von Signifikanzniveaus siehe Fußnote 30.

39 In gleicher Weise entwickeln sich auch die Signifikanzniveaus der Koeffizienten (Einleitungsphase $\mathrm{p}=0,2 ;$ Volksbegehren $\mathrm{p}=0,4 ;$ Volksentscheid $\mathrm{p}=0,45)$.

40 Jann empfiehlt die Verwendung graphischer Verfahren wie Streudiagramme und Leverage-vs.Squared-Residual-Plots sowie die Berechnung von Cooks D, vgl. Ben Jann, Diagnostik von Regressionsschätzern bei kleinen Stichproben, in: Andreas Diekmann (Hrsg.), Methoden der Sozialforschung, Sonderheft 44 der Kölner Zeitschrift für Soziologie und Sozialpsychologie, Wiesbaden 2004, S. 421 - 452. Als Grenzwert für Cooks D wurde D>4/n verwendet, vgl. Ulrich Kohler I Frauke Kreuter, Datenanalyse mit Stata. Allgemeine Konzepte der Datenanalyse und ihre praktische Anwendung, München 2006; Rainer Schnell, Graphisch gestützte Datenanalyse, München 1994.

41 Iwan Barankay / Pascal Sciarini / Alexander Trechsel, a.a.O; Alexander Trechsel, a.a.O; Adrian Vatter, a.a.O.

42 Siehe California Commission on Campaign Financing, a.a.O. 
zeigen sich beträchtliche Unterschiede in der Ausgestaltung dieses direktdemokratischen Instruments, wobei Unterschiede zu bisherigen Volksentscheid-Rankings des Vereins Mehr Demokratie e.V. ${ }^{43}$ vor allen Dingen auf die unterschiedliche Konstruktion der Messinstrumente zurückzuführen sind. Im Gegensatz zu diesen wurde beim Offenheitsindex auf die Inklusion nicht rekonstruierbarer Indikatoren, wie zum Beispiel die Intensität der Reformdebatte, bewusst verzichtet.

Der Offenheitsindex erweist sich als probates Mittel zur Überprüfung des Zusammenhangs zwischen den institutionellen Regelungen der Volksgesetzgebung und ihrer Anwendungshäufigkeit in den einzelnen Bundesländern. Aus den Ergebnissen der Berechnungen lässt sich folgern, dass niedrige Werte auf dem Offenheitsindex nicht gleichbedeutend sind mit geringer Nutzungshäufigkeit, und als Tendenz lässt sich formulieren, dass hohe Werte zu mehr Volksgesetzgebungsverfahren führen können.

Auf Basis der vorliegenden Untersuchung lassen sich komplexere Erklärungsmodelle entwickeln, die neben den Institutionen beispielsweise auch die zunehmende Aufgeschlossenheit der deutschen Parteien gegenüber den direkten Volksrechten ${ }^{44}$ oder die fortschreitende Individualisierung der Bevölkerung ${ }^{45}$ als potentielle Einflussfaktoren berücksichtigen. Ebenso könnte der Erfolgsgrad von Volksgesetzgebungsverfahren einbezogen werden. So ließe sich untersuchen, ob und inwieweit die hier vorgestellten institutionellen Strukturen die Chancen von Anträgen verbessern oder beeinträchtigen.

Ein weiteres Forschungsdesiderat knüpft an so genannte strukturbildende Wirkungen an, die direktdemokratische Institutionen auf das politische System haben können, wie etwa von Leonhard Neidhart im Fall der Schweiz nachgewiesen. ${ }^{46}$ Dort stellen unmittelbare Beteiligungsrechte ein „Verhandlungspfand“ zwischen Regierung und Opposition dar, was zur Inklusion aller „referendumsfähigen Gruppen“ in den Entscheidungsprozess führt. ${ }^{47}$ So soll der Ablehnung von parlamentarischen Entscheidungen durch das Volk vorgebeugt werden.

Die direkte Demokratie in den deutschen Ländern vermag die Kompromissbereitschaft der politischen Akteure zwar zu erhöhen ${ }^{48}$; von einem institutionellen „Konkordanzzwang“ nach Schweizer Muster ${ }^{49}$ zu sprechen, erscheint hingegen unangemessen. Ausgehend vom hier vorgeschlagenen Index könnte jedoch mittels qualitativer Studien untersucht werden, ob mit steigender Offenheit der Volksgesetzgebung auch die Verhandlungsbereitschaft auf Seiten der Parlamentarier wächst.

43 Ralph Kampwirth, a.a.O.; Frank Rehmet / Karin Flothmann / Tim Weber, a.a.O.

44 Siehe Susan E. Scarrow, Party Competition and Institutional Change. The Expansion of Direct Democracy in Germany, in: Party Politics, 3. Jg. (1997), S. $451-472$.

45 Siehe Jürgen Fijalkowski, Erfahrungen mit Volksabstimmungen zu Sachfragen - Erfordernisse und Ergebnisse kategorialer Differenzierung, in: Hans-Dieter Klingemann / Wolfgang Luthardt (Hrsg.), Wohlfahrtsstaat, Sozialstruktur und Verfassungsanalyse, Opladen 1993, S. 147 - 167.

46 Leonhard Neidhart, Plebiszit und pluralitäre Demokratie. Eine Analyse der Funktion des schweizerischen Gesetzesreferendums, Bern 1970.

47 Wolf Linder, Schweizerische Demokratie. Institutionen, Prozesse, Perspektiven, Bern 2005, S. 246.

48 Gunter Jürgens, a.a.O., S. 229.

49 Wolf Linder, a.a.O., S. 246. 